\title{
Relative Attitude Estimation for a Uniform Motion and Slowly Rotating Noncooperative Spacecraft
}

\author{
Liu Zhang, ${ }^{1}$ Zongming Liu, ${ }^{2,3,4}$ Dong Ye, ${ }^{2}$ and Guanyu Zhang ${ }^{1}$ \\ ${ }^{1}$ College of Instrumentation \& Electrical Engineering, Jilin University, Changchun, Jilin 130061, China \\ ${ }^{2}$ School of Electrical Engineering and Automation, Harbin Institute of Technology, Harbin 150001, China \\ ${ }^{3}$ Shanghai Institute of Spaceflight Control Technology, Shanghai 201109, China \\ ${ }^{4}$ Shanghai Key Laboratory of Space Intelligent Control Technology, Shanghai 201109, China
}

Correspondence should be addressed to Guanyu Zhang; zhangguanyu@jlu.edu.cn

Received 6 July 2017; Revised 6 November 2017; Accepted 28 November 2017; Published 27 December 2017

Academic Editor: Alessandro Gasparetto

Copyright (c) 2017 Liu Zhang et al. This is an open access article distributed under the Creative Commons Attribution License, which permits unrestricted use, distribution, and reproduction in any medium, provided the original work is properly cited.

\begin{abstract}
This paper presents a novel relative attitude estimation approach for a uniform motion and slowly rotating noncooperative spacecraft. It is assumed that the uniform motion and slowly rotating noncooperative chief spacecraft is in failure or out of control and there is no a priori rotation rate information. We utilize a very fast binary descriptor based on binary robust independent elementary features (BRIEF) to obtain the features of the target, which are rotational invariance and resistance to noise. And then, we propose a novel combination of single candidate random sample consensus (RANSAC) with extended Kalman filter (EKF) that makes use of the available prior probabilistic information from the EKF in the RANSAC model hypothesis stage. The advantage of this combination obviously reduces the sample size to only one, which results in large computational savings without the loss of accuracy. Experimental results from real image sequence of a real model target show that the relative angular error is about $3.5 \%$ and the mean angular velocity error is about $0.1 \mathrm{deg} / \mathrm{s}$.
\end{abstract}

\section{Introduction}

With the development of the space technology, the number of spacecraft on orbit is increasing rapidly year by year. At the same time, the space debris including thousands of inactive satellites, fragments of broken-up spacecraft, and equipment lost by astronauts is mounting [1]. And there has been a growing interest in autonomously servicing spacecraft on orbit to perform tasks such as spacecraft rescue and repairing, refueling, and removal. Several space programs have been completed or are being developed as technology demonstration of these tasks, such as JAXA's ETS-7 [2], NASA's DART program [3], DARPA's Orbital Express program [4], and SUMO program [5]. On one hand, the malfunctioning spacecraft take up the valuable and limited orbit, which increases the potential security risks. On the other hand, they are also a kind of available resources on orbit. Therefore, the capture of a slowly rotating spacecraft has received detailed attentions in recent years [6-9].
Nowadays, vision-based navigation systems, which have the advantage of being entirely passive and of low power consumption, are usually used to determine the relative attitude and position $[10,11]$. The vision measurement has been separated into two aspects of model based and modelindependent methods [12]. The first class usually needs the CAD model of the target object or some other kinds of a priori knowledge. Cropp et al. [13] extracted the line features in an image by a monocular camera and calculated the matched relationship with a priori model. They got the relative pose of two stable spacecraft by the nonlinear least square optimization theory. The constraint function was the orthogonality between the normal vector of line in the image frame and the plane composed of the same line and the optic center in the camera frame. Kelsey et al. [12] obtained the pose of a slowly rotating spacecraft model by three steps which include the initialization by triangulation, the refinement by Iterative Reweighted Least Squares, and the tracking by EKF. In their 


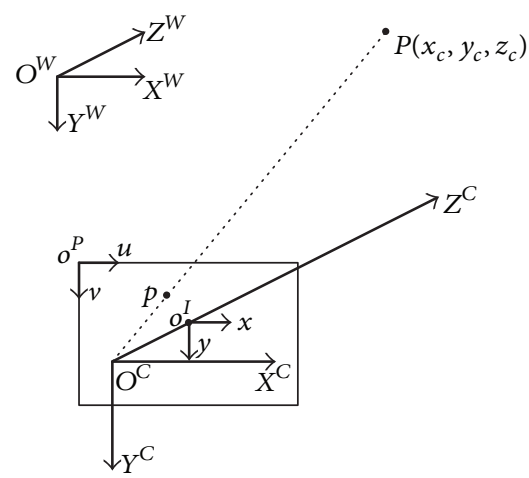

Figure 1: The demonstration of the frames.

experiments in laboratory, the attitude error was up to about 20 degrees and the range error was more than $10 \%$ of range.

The model-independent method is more challenging because no prior information will be used. In other words, the target is absolutely a noncooperative object. Priggemeyer et al. [14] acquired the target picture sequence by a stereo camera system with a baseline of $0.2 \mathrm{~m}$. Afterwards, they extracted the point features with SURF. And then the DLT algorithm [15] solving a linear equation system was applied to find the projection matrices. Finally, a Kalman filter was applied as a recursive least squares filter to reduce noise. Oumer and Panin [16] and Segal et al. [17] presented a system for feature-based 3D tracking of a noncooperative satellite. Sequential 3D motion estimation from a stereo camera has also been tackled using filtering schemes.

Up to now, most of the algorithms and systems are focused on a scene that the camera optical axis is parallel to the spin direction of the target. This hypothesis greatly reduces the degree of difficulty, because the feature face is in the field of view all the way. In our paper, we propose the optical axis and the spin direction, mutually perpendicular. So there is no fixed feature face to our camera. This proposes high demand of the robustness and universality of the algorithm. The contribution of this paper is the proposal of a new single candidate RANSAC algorithm that exploits the probabilistic prediction obtained from the EKF in order to increase the efficiency of the spurious match rejection step. The calculation speed is further improved by using a very fast feature extractor. Meanwhile, we introduce the image pyramid and the binary descriptor for the sake of ensuring the scale invariance and rotational invariance.

\section{Feature Initialization}

2.1. Reference Frames. As in Figure 1, there are some frames defined as follows.

(1) Pixel Frame $\left(O^{p}-u v\right)$. The frame is centered at the top left point of the resolution, the $u$-axis is directed to the right level, and $v$-axis is orthogonal with $u$-axis in the same plane.

(2) Image Frame $\left(O^{I}-x y\right)$. The frame is centered at the place where the optic axis intersects with the image plane; the $x$-axis and $y$-axis are parallel to the $u$-axis and $v$-axis, respectively.

(3) Camera Frame $\left(O^{c}-X^{c} Y^{c} Z^{c}\right)$. The frame is centered at the optic center, $x$-axis is parallel to the $u$-axis, $y$-axis is parallel to the $v$-axis, and $z$-axis is defined as the cross-product of the other two axes.

(4) World Frame $\left(O^{w}-X^{w} Y^{w} Z^{w}\right)$. The frame has its origin and axes anywhere in the real world space.

2.2. Feature Detection and Description. Our proposed feature builds on the well-known fast keypoint detector [18] and the recently developed BRIEF descriptor [19]. Both these techniques are attractive because of their good performance and low cost. FAST takes one parameter, the intensity threshold between the center pixel and those in a circular ring around the center. We use circular radius of 9, which has good performance. FAST does not produce multiscale features. We employ a scale pyramid of the image and produce fast features at each level in the pyramid.

Our approach uses a simple but effective measure of corner orientation, the intensity centroid [20]. Rosin defines the moments of a patch as

$$
m_{p q}=\sum_{u, v} u^{p} v^{q} I(u, v)
$$

where $u$ and $v$ belong to a circular region of radius $r$ in the pixel frame. With these moments, we may find the centroid:

$$
C=\left(\frac{m_{10}}{m_{00}}, \frac{m_{01}}{m_{00}}\right) .
$$

We can construct a vector from the corner's center, $O$, to the centroid, $\overrightarrow{O C}$. The orientation of the patch then simply is

$$
\theta=\arctan \left(\frac{m_{01}}{m_{10}}\right) .
$$

The BRIEF descriptor is a bit string description of an image patch constructed from a set of binary intensity tests. Consider a smoothed image patch, p. A binary test $\tau$ is defined by

$$
\tau(p ; u, v):= \begin{cases}1, & p(u)<p(v) \\ 0, & p(u) \geq p(v),\end{cases}
$$

where $p(\cdot)$ is the intensity of $p$ at a point $(u, v)$. The feature is defined as a vector of $n$ binary tests:

$$
f_{n}(p):=\sum_{1 \leq i \leq n} 2^{i-1} \tau\left(p ; u_{i}, v_{i}\right)
$$

The vector length $n$ could be chosen as 128, 256, 512, and so on. BRIEF has no orientation, so if we want to allow BRIEF to be invariant to in-plane rotation, we should add one to it. Just as described in [21], we use the steered BRIEF operator to solve the problem.

$$
g_{n}(p, \theta):=f_{n}(p) \mid\left(u_{i}, v_{i}\right) \in S_{\theta} .
$$




\section{EKF-Based Estimation}

3.1. State Vector Definition. The whole state vector $X_{k}$ at step $k$ is composed of a set of camera parameters $x_{c}$ and feature set $y$. All of these are referred to by the world frame $W$.

$$
\begin{aligned}
X_{k} & =\left(\begin{array}{c}
x_{c} \\
y
\end{array}\right) \\
P_{k} & =\left(\begin{array}{cc}
P_{c_{k}} & P_{c_{k} y} \\
P_{y c_{k}} & P_{y}
\end{array}\right),
\end{aligned}
$$

where $P_{k}$ is the covariance matrix. The estimated feature set $y$ is composed of $n$ point features $y_{i}, y=\left(y_{1}^{T}, y_{2}{ }^{T}, \ldots, y_{n}{ }^{T}\right)^{T}$. Point features are parametrized in inverse depth coordinate [22] which can be defined by the dimension 6-state vector:

$$
y_{i, I d}=\left(x_{i}, y_{i}, z_{i}, \theta_{i}, \phi_{i}, \rho_{i}\right)^{T} .
$$

The inverse depth parametrization stores in its six parameters the $3 \mathrm{D}$ camera position when the feature was initialized $\left(x_{i}, y_{i}, z_{i}\right)^{T}$, the azimuth-elevation pair $\left(\theta_{i}, \phi_{i}\right)^{T}$ encoding the unit ray pointing to the feature, and its inverse depth along the ray $\rho$ [23]. They could be converted to Euclidean $X Y Z$ coordinates $y_{i, E}=\left(X_{i}, Y_{i}, Z_{i}\right)^{T}$ if and when the projection equation becomes linear enough.

$$
y_{i, E}=\left(\begin{array}{c}
X_{i} \\
Y_{i} \\
Z_{i}
\end{array}\right)=\left(\begin{array}{c}
x_{i} \\
y_{i} \\
z_{i}
\end{array}\right)+\frac{1}{\rho_{i}} m\left(\theta_{i}, \phi_{i}\right),
$$

where $m\left(\theta_{i}, \varphi_{i}\right)=\left(\cos \phi_{i} \sin \theta_{i},-\sin \phi_{i}, \cos \phi_{i} \cos \theta_{i}\right)^{T}$.

3.2. Dynamic Model. The camera state $x_{c}$ is composed of pose terms: $r^{W C}$ camera optical center position and $\sigma^{W C}$ modified Rodrigues parameters (MRPs) defining orientation; linear and angular velocity $v^{W}$ and $\omega^{C}$ relative to world frame $W$ and camera frame $C$.

The state update equation for the camera is

$$
f_{v}=\left(\begin{array}{c}
r_{k+1}^{W C} \\
\sigma_{k+1}^{W C} \\
v_{k+1}^{W} \\
\omega_{k+1}^{C}
\end{array}\right)=\left(\begin{array}{c}
r_{k}^{W C}+\left(v_{k}^{W}+V_{k}^{W}\right) \Delta t \\
\frac{1}{4}\left[\left(1-\sigma_{k}^{W C^{T}} \sigma_{k}^{W C}\right) I+2\left[\sigma_{k}^{W C} \times\right]+2 \sigma_{k}^{W C} \sigma_{k}^{W C^{T}}\right]\left(\omega_{k}^{C}+\Omega^{C}\right) \Delta t \\
v_{k}^{W}+V^{W} \\
\omega_{k}^{C}+\Omega^{C}
\end{array}\right)
$$

where

$$
\left[\sigma_{k}^{W C} \times\right]=\left[\begin{array}{ccc}
0 & -\sigma_{k z}^{W C} & \sigma_{k y}^{W C} \\
\sigma_{k z}^{W C} & 0 & -\sigma_{k x}^{W C} \\
-\sigma_{k y}^{W C} & \sigma_{k x}^{W C} & 0
\end{array}\right]
$$

$v^{W}$ and $\Omega^{C}$ are zero-mean Gaussianly distributed velocity noise coming from an impulse of acceleration.

3.3. Measurement Model. The measurement model used in the experiments of the paper is a pinhole camera model plus a two-parameter radial distortion. The camera is assumed to be calibrated in advance. Inverse depth and Euclidean points in the state vector are first transformed to the camera reference frame:

$$
h^{C}=\left(\begin{array}{c}
h_{x} \\
h_{y} \\
h_{z}
\end{array}\right)=h_{i, E}=R^{C W}\left(\begin{array}{c}
X_{i} \\
Y_{i}-r^{W C} \\
Z_{i}
\end{array}\right)
$$

$$
\begin{aligned}
h^{C} & =\left(\begin{array}{l}
h_{x} \\
h_{y} \\
h_{z}
\end{array}\right)=h_{i, I d} \\
& =R^{C W}\left(\rho_{i}\left(\left(\begin{array}{c}
x_{i} \\
y_{i} \\
z_{i}
\end{array}\right)-r^{W C}\right)+m\left(\theta_{i}, \varphi_{i}\right)\right),
\end{aligned}
$$

where $R^{C W}$ represents a rotation matrix computed from the state MRPs and $m\left(\theta_{i}, \varphi_{i}\right) m$ is the function converting azimuth-elevation angles to a unit vector. Points in the camera frame are then projected using the standard pinhole model:

$$
h_{u}=\left(\begin{array}{l}
u \\
v
\end{array}\right)=\left(\begin{array}{c}
u_{0}-\frac{f}{d x} \frac{h_{x}}{h_{z}} \\
v_{0}-\frac{f}{d y} \frac{h_{y}}{h_{z}}
\end{array}\right) .
$$

Here $f$ stands for the focal length of the camera, $d x$ and $d y$ represent the pixel size, and $\left(u_{0}, v_{0}\right)^{T}$ are the image center coordinates. The imaged point is finally transformed using 
the two parameters $k_{1}, k_{2}$, modeled below, resulting in the distorted measurement

$$
\begin{aligned}
& h_{d}=\left(\begin{array}{l}
u_{d} \\
v_{d}
\end{array}\right)=\left(\begin{array}{c}
u_{0}+\frac{u-u_{0}}{1+k_{1} r_{d}^{2}+k_{2} r_{d}^{4}} \\
v_{0}+\frac{v-v_{0}}{1+k_{1} r_{d}^{2}+k_{2} r_{d}^{4}}
\end{array}\right) \\
& r_{u}=\sqrt{\left(d x\left(u-u_{0}\right)\right)^{2}+\left(d y\left(v-v_{0}\right)\right)^{2}} \\
& r_{u}=r_{d}\left(1+k_{1} r_{d}^{2}+k_{2} r_{d}^{4}\right) .
\end{aligned}
$$

\section{Single Candidate RANSAC for EKF Estimation}

In the case of the new single candidate RANSAC presented here, extra information for the camera motion comes from the probability distribution function that the EKF naturally propagates over time. Computational savings with respect to standard RANSAC [24] can be easily derived. The number of RANSAC random hypotheses $n_{\text {hyp }}$ necessary to guarantee that at least one of them is mismatch-free with probability $\mu$ can be computed using the following formula:

$$
n_{\text {hyp }}=\frac{\log (1-\mu)}{\log \left(1-(1-\varepsilon)^{m}\right)},
$$

where $\varepsilon$ is the assumed inlier ratio and $m$ the number of measurements that instantiate the model. As a simple but illustrative example, if the inlier ratio $\varepsilon$ is 0.4 and the probability $\mu$ equals 0.99 , the number of random hypotheses would be reduced from 447 for $m=5$ to only 9 for $m=1$. The efficiency is highly improved. The update is performed in two steps using the standard extended Kalman filter equations.

Firstly, we will find the matching relationship between the point features estimated from those in the last image and those extracted in the current one. The inliers in the current image are represented by $z_{I C}=\left\{z_{1}, z_{2}, \ldots, z_{i}, \ldots, z_{n}\right\}$.

Next, we could update the state vector $x_{i}$ by picking up one point $z_{i}$ from the set of $z_{\text {IC }}$ randomly.

$$
\begin{aligned}
S_{k+1} & =H_{k+1} P_{k+1 \mid k} H_{k+1}^{T}+R_{k+1} \\
K_{k+1} & =P_{k+1 \mid k} H_{k+1}^{T} S_{k+1}^{-1} \\
\widehat{x}_{i} & =\widehat{x}_{k+1 \mid k}+K_{k+1}\left(z_{i}-h_{k}\right) \\
P_{k+1} & =\left(I-K_{k+1} H_{k+1}\right) P_{k+1 \mid k} .
\end{aligned}
$$

Consequently we get a new $z_{\text {IC }}$ set, and then we finally update the EKF state and covariance.

$$
\begin{aligned}
K_{k+1} & =P_{k+1 \mid k} H_{k+1}^{T}\left(H_{k+1} P_{k+1 \mid k} H_{k+1}^{T}+R_{k+1}\right)^{-1} \\
\widehat{x}_{k+1} & =\widehat{x}_{k+1 \mid k}+K_{k+1}\left(z_{i, \mathrm{NEW}}-h_{k}\right) \\
P_{k+1} & =\left(I-K_{k+1} H_{k+1}\right) P_{k+1 \mid k} .
\end{aligned}
$$

The initial measurement for a newly observed feature inserted into the state vector is

$$
f\left(\widehat{r}^{W C}, \widehat{q}^{W C}, h, \rho_{0}\right)=\left(\widehat{x}_{i}, \widehat{y}_{i}, \widehat{z}_{i}, \widehat{\theta}_{i}, \widehat{\phi}_{i}, \widehat{\rho}_{i}\right)^{T},
$$

where $\widehat{r}^{W C}, \widehat{q}^{W C}$ represent the current camera pose estimate, $h=(u, v)^{T}$ is the image observation, and $\rho_{0}$ is the depth prior. In (17),

$$
\begin{aligned}
\widehat{r}^{W C} & =\left(\widehat{x}_{i}, \widehat{y}_{i}, \widehat{z}_{i}\right)^{T} \\
h^{W} & =R_{W C}\left(\widehat{\sigma}^{W C}\right)(u, v, \mathbf{1})^{T},
\end{aligned}
$$

where $u$ and $v$ are normalized retina image coordinates. Despite $h^{W}$ being a non-unit directional vector, the angles by which we parametrize its direction can be calculated as

$$
\left(\begin{array}{c}
\theta_{i} \\
\phi_{i}
\end{array}\right)=\left(\begin{array}{c}
\arctan \left(h_{x}^{W}, h_{z}^{W}\right) \\
\arctan \left(-h_{Y}^{W}, \sqrt{h_{x}^{W^{2}}+h_{z}^{W^{2}}}\right)
\end{array}\right) .
$$

The covariance of $\widehat{x}_{i}, \widehat{y}_{i}, \widehat{z}_{i}, \widehat{\theta}_{i}$, and $\widehat{\phi}_{i}$ is derived from the image measurement error covariance $R_{i}$ and the state covariance estimate $\widehat{P}_{k \mid k}$. We set $\widehat{\rho}_{\mathbf{0}}=\mathbf{0 . 1}$ and $\sigma_{\rho}=\mathbf{0 . 5}$.

The state covariance after feature initialization is

$$
\begin{aligned}
& \widehat{P}_{k \mid k}^{\text {new }}=J\left(\begin{array}{ccc}
\widehat{P}_{k \mid k} & \mathbf{0} & \mathbf{0} \\
\mathbf{0} & R_{i} & \mathbf{0} \\
\mathbf{0} & \mathbf{0} & \sigma_{i}^{2}
\end{array}\right) J^{T}
\end{aligned}
$$

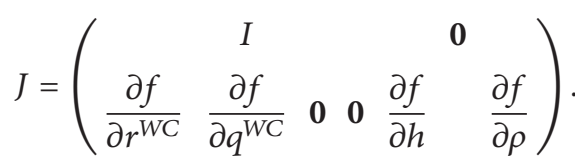

After each estimation step, the linearity index

$$
L_{d}=\left|\frac{\left(\left(\partial^{2} u / \partial d^{2}\right) 2 \sigma_{d}\right)}{\partial u / \partial d}\right|=\frac{4 \sigma_{d}}{d_{1}}|\cos \alpha|
$$

is computed for every feature coded in inverse depth:

$$
\begin{aligned}
h_{x y z}^{W} & =\widehat{x}_{i}-\widehat{r}^{W C}, \\
\sigma_{d} & =\frac{\sigma_{\rho}}{\rho_{i}^{2}}, \quad \sigma_{\rho}=\sqrt{P y_{i} y_{i}(\mathbf{6}, \mathbf{6})}, \\
d_{i} & =\left\|h_{x y z}^{W}\right\|, \\
\cos \alpha & =m^{T} h_{x y z}^{W}\left\|h_{x y z}^{W}\right\|^{-1},
\end{aligned}
$$

where $\widehat{x}_{i}$ is computed using (9) and $P y_{i} y_{i}$ is the submatrix $6 * 6$ covariance matrix or the corresponding considered feature. The full state covariance matrix $P$ is transformed with the corresponding Jacobian:

$$
\begin{aligned}
P_{\text {new }} & =J P J^{T} \\
J & =\operatorname{diag}\left(I, \frac{\partial x_{i}}{\partial y_{i}}, I\right) .
\end{aligned}
$$




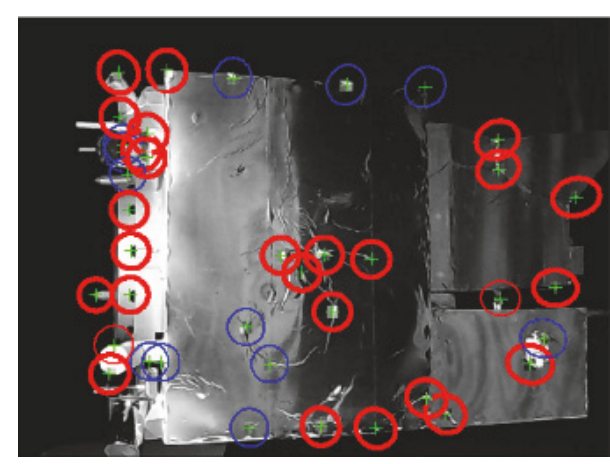

(a)

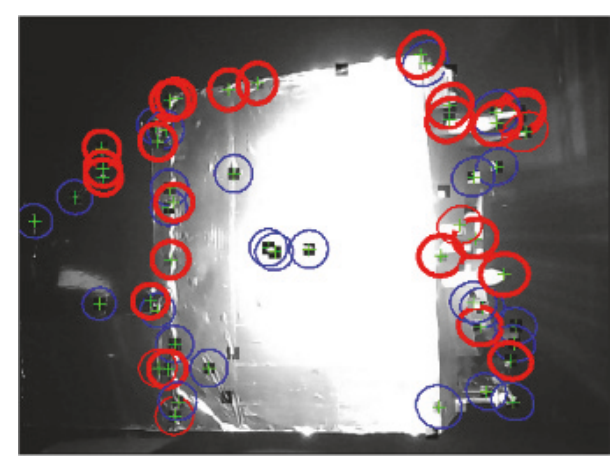

(c)

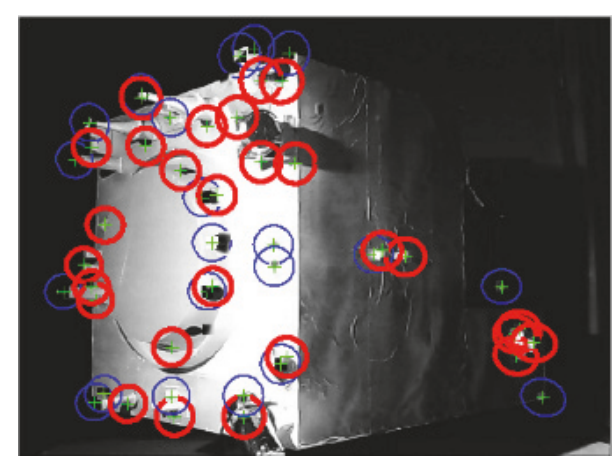

(b)

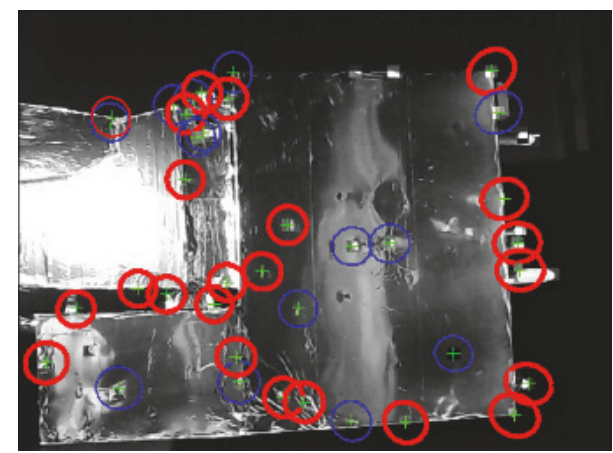

(d)

FIGURE 2: Example of images taken from the monocular sequence.

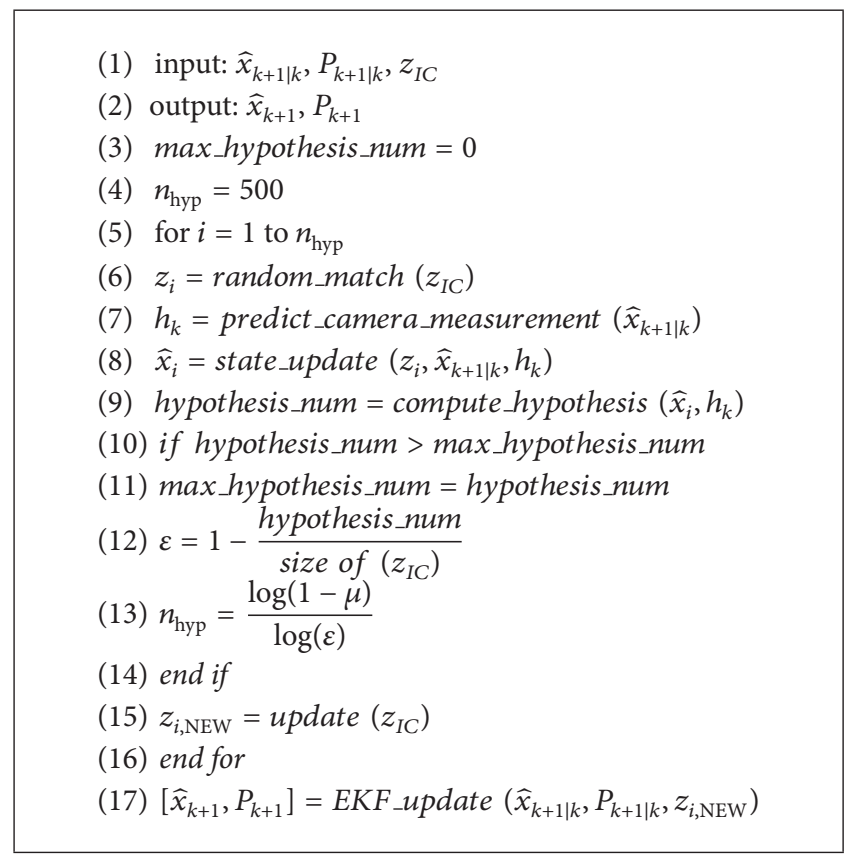

Algorithm 1: The algorithm flow.

In Algorithm 1, a pseudocode is shown to state the core steps of single candidate RANSAC for EKF estimation.

\section{Experiment Results}

The performance of our algorithm has been tested on real image sequences acquired with a monocular camera under space lighting conditions, based on a terrestrial simulation mockup. The experiment was carried out with a real size model and a camera with a $40^{\circ}$ field of view and $640 \times 480$ resolution, capturing monochrome image sequences at $10 \mathrm{fps}$. In the end of a dark room, the model covered with a reflective golden foil was mounted on a swivel table which operated at the speed of $2 \mathrm{deg} / \mathrm{s}$ around the vertical direction. And on the other side, the camera was fixed on a 3-degree-of-freedom rail. A direct high-power floodlight, simulating the spectrum of the sun, illuminates the surface of the model at different incident angles.

Figures 2(a)-2(d) show a spaceflight mockup from different view with the red circles on behalf of the tracked features and the blue ones representing the lost ones. Figures 3 and 4 display the equivalent trajectory of the camera in 2 dimensions and 3 dimensions, respectively. Because the camera was fixed in front of the mockup, the trajectory is almost round. Because the satellite is rotating continuously, the measured angle changes periodically. Figure 5 correctly exhibits the situation. There are two lines in the left column in Figure 6: the blue dotted one stands for the actual value, while the red one represents the estimated value. Meanwhile, the absolute error and relative error are shown in Figure 7. 

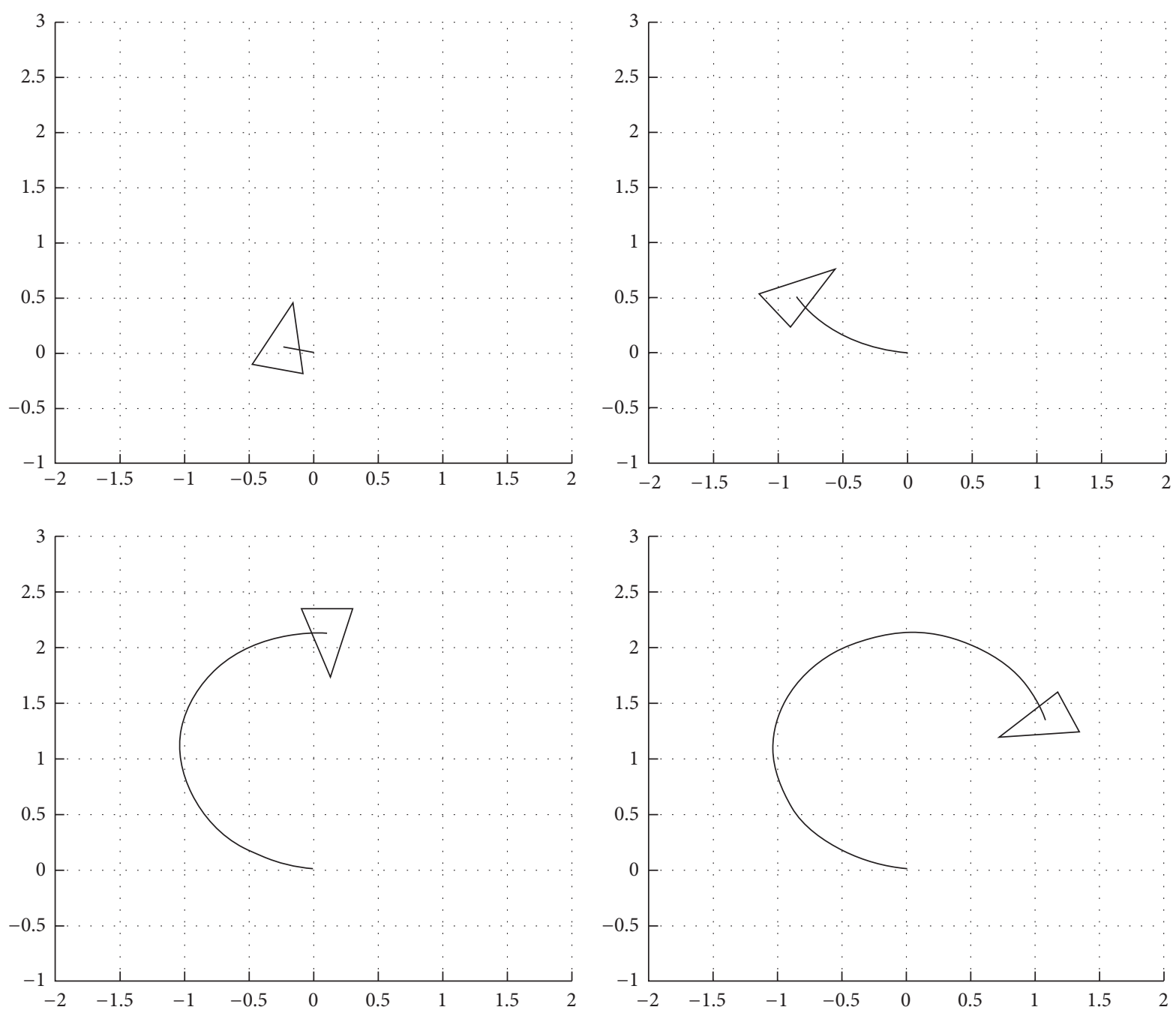

FIgURE 3: The corresponding equivalent trajectory of the camera in 2 dimensions.

We present 3 cycles. Because we use Euler angle to express the rotation, the maximum degree is $180 \mathrm{deg}$. And the jump points just show the phase switches. If we use the angular accumulation to express the rotation, there will be no jump points. We present here a fitting function using the angular dataset and the image sequence, based on linear polynomial regression algorithm.

$$
f(t)=0.2073 t+0.1953,
$$

where $t$ is the number of the frame in image sequence and $f(t)$ represents the corresponding angle. Through analysis, it is pointed out that the mean error between actual spinning rate and the estimated is below $0.1 \mathrm{deg} / \mathrm{s}$.

\section{Conclusions}

In this paper, we presented a system for feature-based 3D tracking of a noncooperative spaceflight. The rotational angle and velocity of the target are estimated in camera frame, using image-plane motion fields. Preliminary experimental results have been obtained from a simulation mockup, demonstrating robustness and accuracy properties of the proposed method. The rigid motion is estimated by a bank of single candidate RANSAC EKF which greatly promotes the efficiency of computation. With the combination of scale and rotational invariance feature extractor, the system becomes more robust.

Future system improvements will be the integration of a laser and a camera or two cameras to obtain the depth information, possibly including different features (points, vanish points, lines, planes, etc.), and introducing loop-closure mechanisms and bag of words to improve the accuracy and stability.

\section{Conflicts of Interest}

The authors declare that they have no conflicts of interest.

\section{Acknowledgments}

The research was sponsored by Shanghai Rising-Star Program (no. 16QB1401000), Key Project of Shanghai Science and 

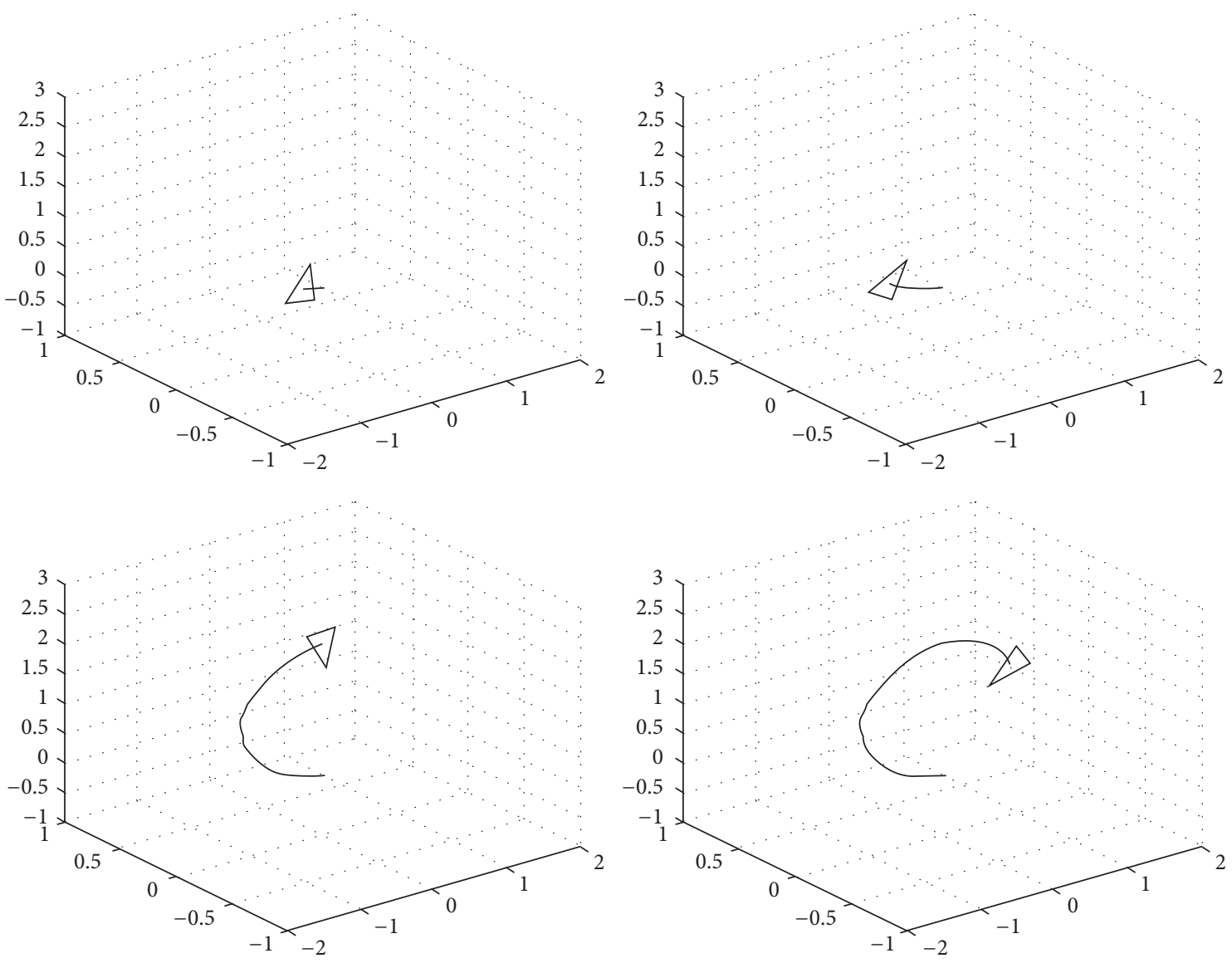

FIgURE 4: The corresponding equivalent trajectory of the camera in 3 dimensions.

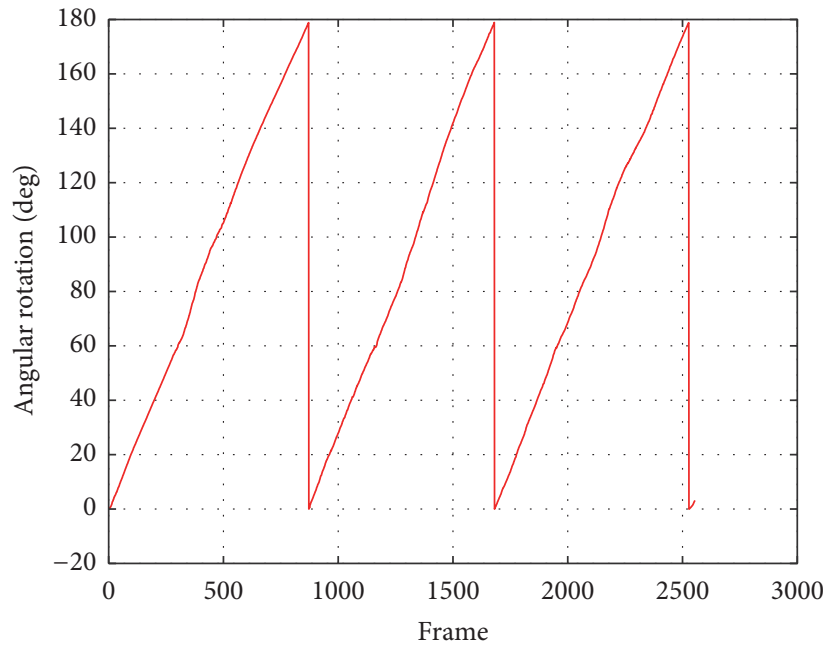

FIgURE 5: The curve of the rotating angular estimation. 


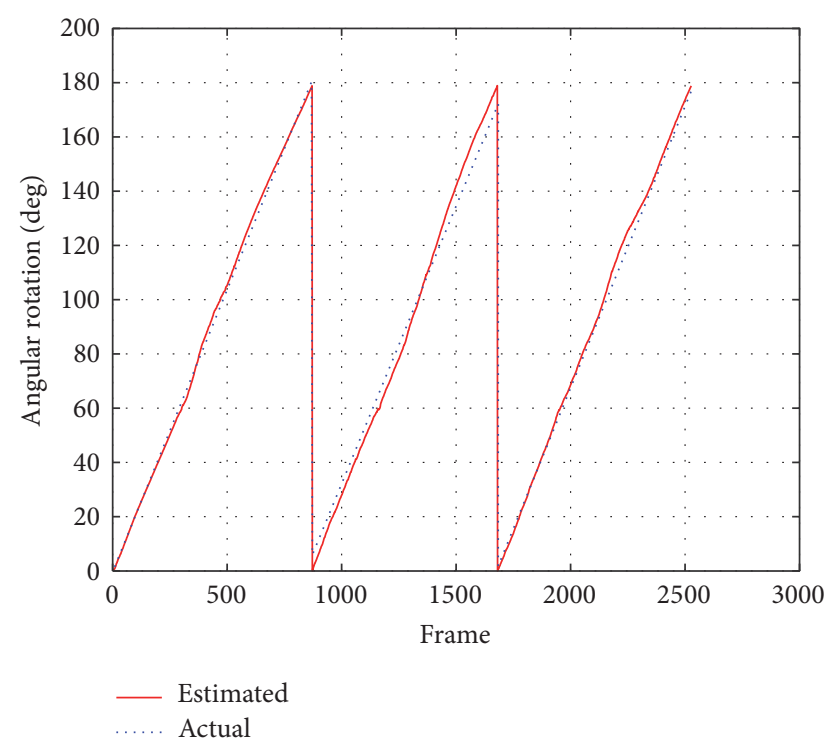

Figure 6: Actual and estimated curves.
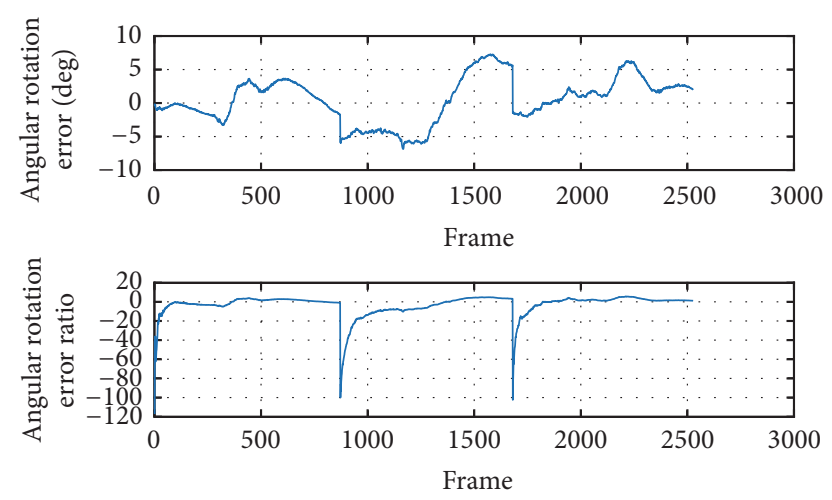

FIGURE 7: Estimation results for the experiment.

Technology Committee (no. 16DZ1120400), and the National Natural Science Foundation of China (Project no. 51705187).

\section{References}

[1] A. M. Long, M. G. Richards, and D. E. Hastings, "On-orbit servicing: a new value proposition for satellite design and operation," Journal of Spacecraft and Rockets, vol. 44, no. 4, pp. 964976, 2007.

[2] I. Kawano, M. Mokuno, T. Kasai, and T. Suzuki, "Result of autonomous rendezvous docking experiment of engineering test satellite. VII," Journal of Spacecraft and Rockets, vol. 38, no. 1, pp. 105-111, 2001.

[3] M. Braukus and K. Newton, "On orbit anomaly ends DART mission early," updated April 2005, http://www.nasa.gov/mission_ pages/dart/media/05-051.html.

[4] D. A. Whelan, E. A. Adler, S. B. Wilson, and G. Roesler, "DARPA orbital express program: effecting a revolution in space-based systems," in International Symposium on Optical Science and Technology, vol. 4136 of Proceedings of SPIE, pp. 48-56, The International Society for Orbital Engineering, San Diego, Calif, USA, 2000.
[5] A. B. Bosse, W. J. Barnds, M. A. Brown et al., "SUMO: spacecraft for the universal modification of orbits," in Defense and Security Symposium, vol. 5419 of Proceedings of SPIE, pp. 36-46, Orlando, Fla, USA, 2004

[6] F. Aghili and K. Parsa, "Motion and parameter estimation of space objects using laser-vision data," Journal of Guidance, Control, and Dynamics, vol. 32, no. 2, pp. 537-549, 2009.

[7] S.-I. Nishida and S. Kawamoto, "Strategy for capturing of a tumbling space debris," Acta Astronautica, vol. 68, no. 1-2, pp. 113-120, 2011.

[8] J. K. Thienel and R. M. Sanner, "Hubble space telescope angular velocity estimation during the robotic servicing mission," Journal of Guidance, Control, and Dynamics, vol. 30, no. 1, pp. 29-34, 2007.

[9] M. Xin and H. Pan, "Nonlinear optimal control of spacecraft approaching a tumbling target," Aerospace Science and Technology, vol. 15, no. 2, pp. 79-89, 2011.

[10] S. G. Kim, J. L. Crassidis, Y. Cheng, A. M. Fosbury, and J. L. Junkins, "Kalman filtering for relative spacecraft attitude and position estimation," Journal of Guidance, Control, and Dynamics, vol. 30, no. 1, pp. 133-143, 2007.

[11] Y. Xing, X. Cao, S. Zhang, H. Guo, and F. Wang, "Relative position and attitude estimation for satellite formation with coupled translational and rotational dynamics," Acta Astronautica, vol. 67, no. 3-4, pp. 455-467, 2010.

[12] J. M. Kelsey, J. Byrne, M. Cosgrove, S. Seereeram, and R. K. Mehra, "Vision-based relative pose estimation for autonomous rendezvous and docking," in Proceedings of the IEEE Aerospace Conference, March 2006.

[13] A. Cropp, P. Palmer, and C. I. Underwood, "Pose estimation of target satellite for proximity operations," in Proceedings of the Small Satellite Conference, 2000.

[14] M. Priggemeyer, M. Dimartino, and J. Rossmann, "Pose estimation and trajectory calculation for autonomous close-range rendezvous and docking," in Proceedings of the IEEE/ASME International Conference on Advanced Intelligent Mechatronics, pp. 15-20, July 2015.

[15] R. Hartley and A. Zisserman, Multiple View Geometry in Computer Vision, Cambridge University Press, 2nd edition, 2000.

[16] N. W. Oumer and G. Panin, "Tracking and pose estimation of non-cooperative satellite for on-orbit servicing," in Proceedings of the International Symposium on Artificial Intelligence, Robotics and Automation in Space, 2012.

[17] S. Segal, A. Carmi, and P. Gurfil, "Vision-based relative state estimation of non-cooperative spacecraft under modeling uncertainty," in Proceedings of the IEEE Aerospace Conference, pp. 1-8, March 2011.

[18] E. Rosten and T. Drummond, "Machine learning for high speed corner detection," in Proceedings of the European Conference on Computer Vision, pp. 430-443, 2006.

[19] M. Calonder, V. Lepetit, C. Strecha, and P. Fua, "Brief: binary robust independent elementary features," in Proceedings of the European Conference on Computer Vision, pp. 778-792, 2010.

[20] P. L. Rosin, "Measuring corner properties," Computer Vision and Image Understanding, vol. 73, no. 2, pp. 291-307, 1999.

[21] E. Rublee, V. Rabaud, K. Konolige, and G. Bradski, "ORB: an efficient alternative to SIFT or SURF," in Proceedings of the IEEE International Conference on Computer Vision (ICCV '11), pp. 2564-2571, Barcelona, Spain, November 2011.

[22] J. Civera, A. J. Davison, and J. M. M. Montiel, "Inverse depth parametrization for monocular SLAM," IEEE Transactions on Robotics, vol. 24, no. 5, pp. 932-945, 2008. 
[23] J. Civera, O. G. Grasa, A. J. Davison, and J. M. M. Montiel, "1Point RANSAC for extended Kalman filtering: application to real-time structure from motion and visual odometry," Journal of Field Robotics, vol. 27, no. 5, pp. 609-631, 2010.

[24] D. Nistér, "An efficient solution to the five-point relative pose problem," IEEE Transactions on Pattern Analysis and Machine Intelligence, vol. 26, no. 6, pp. 756-770, 2004. 


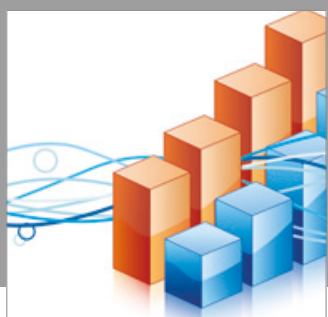

Advances in

Operations Research

vatersals

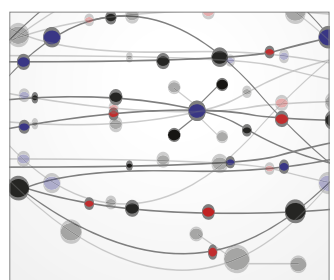

\section{The Scientific} World Journal
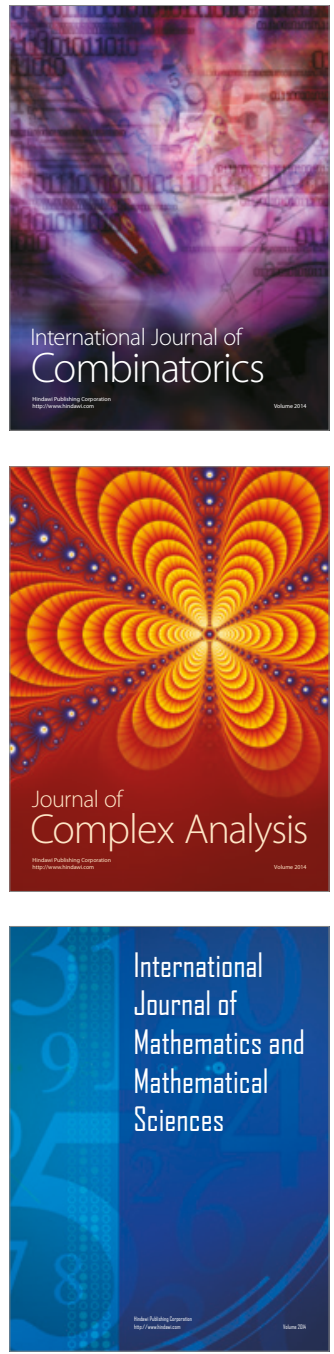
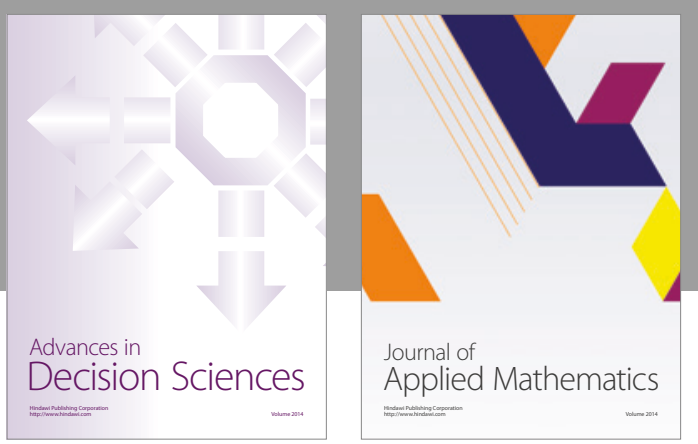

Algebra

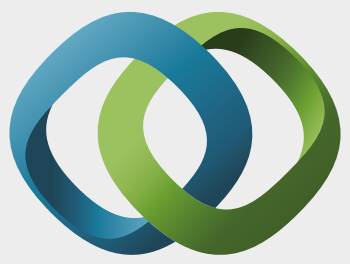

\section{Hindawi}

Submit your manuscripts at

https://www.hindawi.com
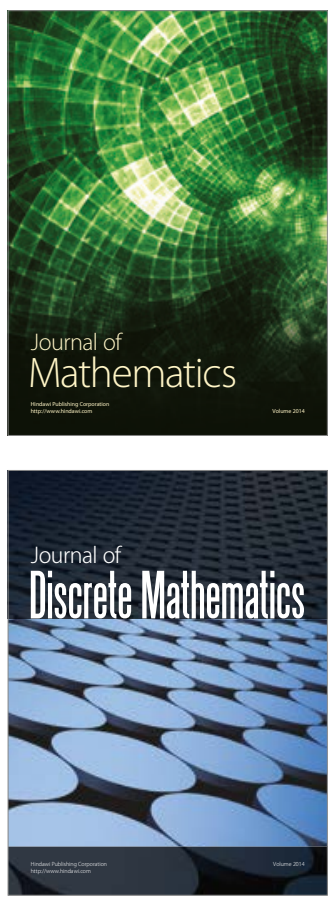

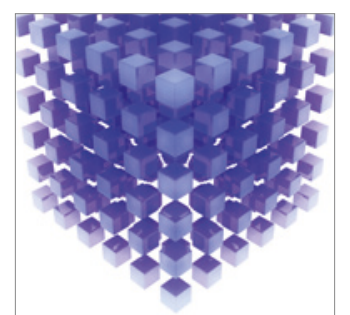

Mathematical Problems in Engineering
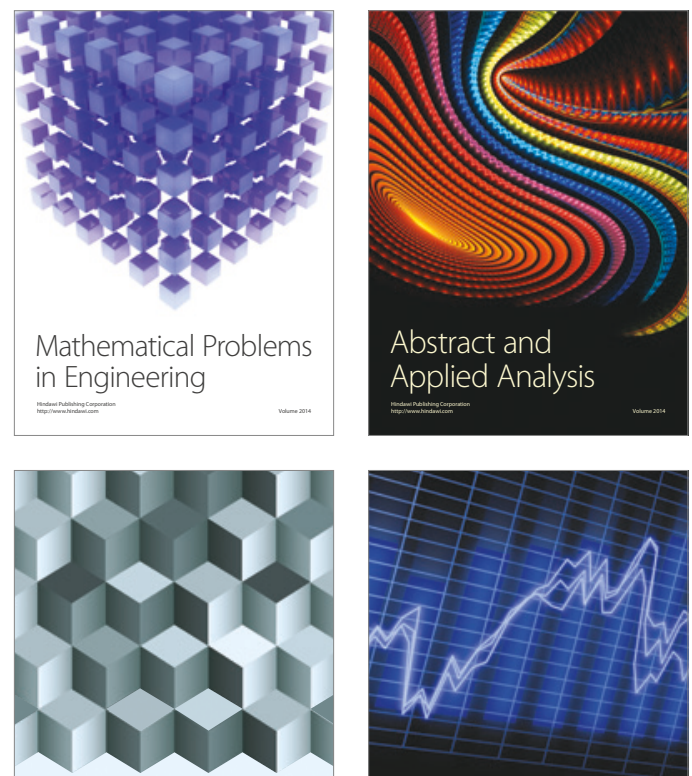

Journal of

Function Spaces

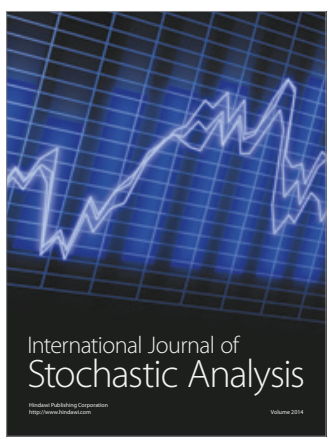

Probability and Statistics
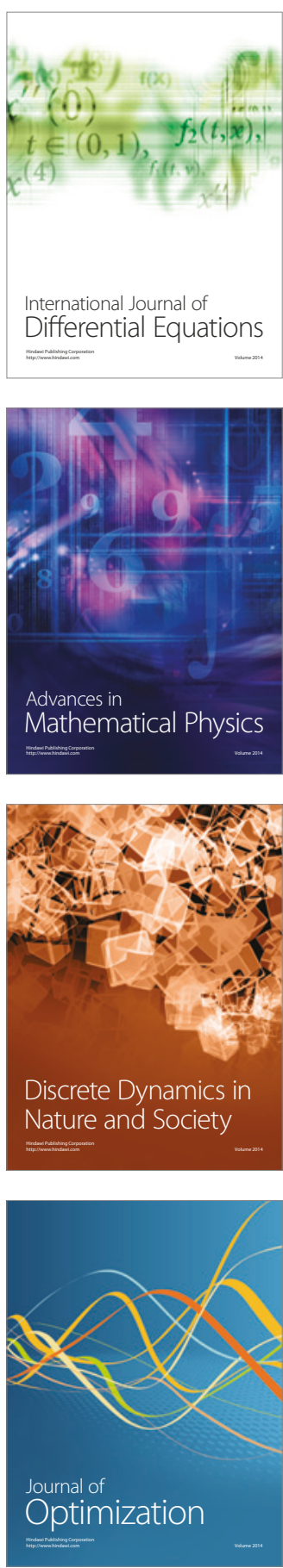